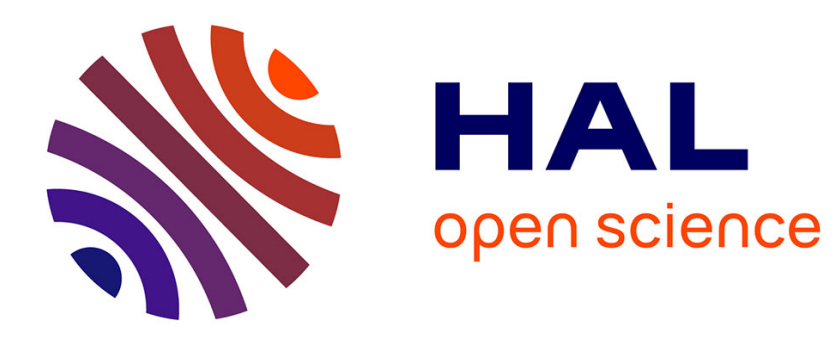

\title{
Mohsen Kadivar, un clerc militant et réformiste
} Sabrina Mervin

\section{- To cite this version:}

Sabrina Mervin. Mohsen Kadivar, un clerc militant et réformiste. Sabrina Mervin. Les mondes chiites et l'Iran, Karthala-IFPO, pp.417-430, 2007, 9782845868885. halshs-01858567

\section{HAL Id: halshs-01858567 https://shs.hal.science/halshs-01858567}

Submitted on 23 Aug 2018

HAL is a multi-disciplinary open access archive for the deposit and dissemination of scientific research documents, whether they are published or not. The documents may come from teaching and research institutions in France or abroad, or from public or private research centers.
L'archive ouverte pluridisciplinaire HAL, est destinée au dépôt et à la diffusion de documents scientifiques de niveau recherche, publiés ou non, émanant des établissements d'enseignement et de recherche français ou étrangers, des laboratoires publics ou privés. 
Article publié dans Sabrina Mervin (dir.), Les mondes chiites et l'Iran, Paris, Karthala-IFPO, 2007, pp. 417-430.

\section{Mohsen Kadivar, un clerc militant et réformiste par Sabrina Mervin}

Depuis une dizaine d'années, Mohsen Kadivar s'est taillé une place de choix dans le champ des penseurs réformistes iraniens où chacun doit se distinguer par sa démarche, sa méthode, par les sujets qu'il aborde, et par son talent d'auteur et d'orateur, car si ces milieux sont très policés, la concurrence y est rude. En outre, les réformistes iraniens sont souvent présentés dans une perspective comparative, mis les uns au regard des autres, et ils contribuent parfois à des ouvrages collectifs qui les rassemblent ${ }^{1}$. Né en 1959, Mohsen Kadivar n'est pas un «intellectuel religieux », mais un clerc doté d'une solide formation en sciences religieuses, un pur produit de la hozeh de Qom et de la révolution islamique. En tant que clerc, il revendique de réfléchir sur le monde qui l'entoure. Aussi, pour lui, le problème de la production intellectuelle en Iran est simple : " Nos traditionnels connaissent très peu, ou pas du tout, les exigences du monde moderne, et nos modernistes n'ont pas de connaissance approfondie de leur tradition (sonnat), de leur culture et de leur religion.» Ceux qui connaissent à la fois la religion et le monde sont donc investis d'une mission, affirmer et prouver à al-Azhar, à Najaf et à Qom que la perception de la rationalité a changé depuis la Renaissance (en français dans le texte) ${ }^{2}$. Partant de l'idée que c'est à partir de la tradition que l'on construit une modernité endogène, Kadivar critique les doctrines et les institutions de l'islam, comme les principes fondateurs de la République islamique, de l'intérieur. C'est dire que, contrairement à Mojtahed Shabestari, qui est aussi un clerc, il reste dans les sources islamiques, particulièrement le feqh, et ne se réfère ni aux théologiens chrétiens, ni aux sciences sociales.

Mohsen Kadivar est un clerc militant puisqu'il s'engage en politique ; c'est en écrivant sur la philosophie politique chiite et les droits humains en islam qu'il s'est fait connaître. Il s'est peu à peu attaqué à des sujets aussi cruciaux que sensibles, en radicalisant ses positions réformistes, au fur et à mesure d'une manière de désenchantement à l'égard de la révolution islamique. Il fut sans doute par ailleurs influencé par son maître, l'ayatollâh Ali Montazeri, lui-même devenu, selon l'expression de Wilfried Buchta, « la conscience de la révolution » ${ }^{3}$,

\footnotetext{
${ }^{1}$ Voir, par exemple, Farzin V., "Post-revolutionary Discourse of Mohammad Mojtahed Shabestari and Mohsen », Critique, 16 et 17 (2000); Sadri, M., "Sacral Defense of Secularism : The Political Theologies of Soroush, Shabestari and Kadivar», International Journal of Politics, Culture and Society, 15/2, hiver 2001, pp. 257-270.

${ }^{2}$ Conférence sur les droits de la femme donnée au parti Moshârekat à Téhéran, le 15/6/2003. Cf. www.kadivar.com.

${ }^{3}$ Buchta, W., Who rules Iran, p. 92.
} 
par des prises de positions critiques vis-à-vis du gouvernement qui lui valurent une longue mise à l'écart. Mohsen Kadivar, lui, a écrit un traité sur la pensée politique en islam qui se divise en deux volumes : le premier est un exposé sur les diverses théories proposées par les savants chiites, qu'il a classées en neuf catégories ${ }^{4}$; le second est une analyse critique de celle de Khomeini, velâyat-e faqih. L'auteur estime en conclusion que cette théorie ne fait pas partie des fondements du chiisme, mais qu'elle n'est qu'une hypothèse mineure en matière de droit islamique ${ }^{5}$.

À l'issue de cet essai, l'auteur prône une approche démocratique du gouvernement, qu'il ne voit pas réservée aux sociétés non religieuses, mais tout à fait applicable aux sociétés musulmanes ${ }^{6}$. Paradoxe de la République islamique, l'ouvrage fut publié en 1998 et réédité plusieurs fois depuis. Kadivar fut néanmoins condamné à dix-huit mois de prison, suite à une conférence à Ispahan où il récusait la justification religieuse de la terreur politique et l'assassinat d'intellectuels, et à un entretien à la revue Khordad où il reprochait à la République islamique son incapacité à garantir la sécurité de ses citoyens ${ }^{7}$. Il fut relâché en février 2000. Son séjour à la prison d'Evin avait renforcé sa popularité et l'avait conforté sur la voie réformiste. Il orienta ses réflexions sur les droits humains avant de revenir à la philosophie politique, avec une étude sur l'un des pères du mouvement constitutionnaliste du début du XX ${ }^{\mathrm{e}}$ siècle, en Iran, Mollâ Kâzem Khorasâni.

Selon l'usage à la hozeh, Mohsen Kadivar a commencé à enseigné tôt, lorsqu'il était étudiant en sciences religieuses. Ensuite, il a donné des cours de philosophie dans plusieurs universités à Téhéran, dont Tarbiyat-e modarres, où il est encore aujourd'hui. Sa renommée à l'extérieur lui a permis d'être invité dans des universités japonaises et à Harvard, où il a assuré un séminaire, en 2002, sur la raison dans l'appareil méthodologique du droit islamique chiite. Toutefois, sa pensée est moins diffusée, hors d'Iran, que celle de Soroush ou même de Shabestari. Il nous livre ici son parcours intellectuel.

S.M.

- Comment vous êtes-vous orienté vers les études religieuses?

- À l'époque où je fréquentais l'université, j'étais influencé par le docteur Shariati et souhaitais me tourner vers la sociologie, mais ma famille refusa. Je m'inscrivis en ingénierie électronique où j'étudiai un an, puis l'université ferma et, un an plus tard, la révolution culturelle commença. Je me mis alors à l'étude des sciences islamiques à Shirâz mais, au bout

\footnotetext{
${ }^{4}$ Elles sont énoncées dans l'article de Sadri (2001), M., pp. 263-264.

${ }^{5}$ Cf. citation, ibid., p. 265.

${ }^{6}$ On trouvera un résumé de sa pensée sur ces questions dans «Velayat-e faqih and Democracy », www.kadivar.com

7 Mottaghi, M., Iran: les nouveaux intellectuels musulmans, thèse de doctorat inédite, EHESS, 2004, p. 307. L'acte d'accusation et le plaidoyer de Kadivar sont publiés dans Kadivar, M., « Bahâ-ye azade » (Le prix de la libérté), nashr-e nay, 1999.
} 
d'un an, m'étant rendu compte que le niveau des professeurs n'était pas très élevé, je suis parti pour Qom.

La révolution aida. C'est-à-dire que si la révolution n'était pas arrivée, je pense que je ne me serais pas engagé dans cette voie... À Qom, j'ai recommencé le cursus à partir du début car je voulais acquérir des bases solides.

Les dix premières années que j'ai passées à la hozeh, j'ai vraiment essayé d'étudier de manière traditionnelle à tel point que je me suis rapproché de professeurs qui avaient peu de liens avec la politique. L'un d'entre eux m'a dit qu'il n'avait pas lu de journaux depuis trente ans.

- Vous aviez donc opté pour le système traditionnel de la hozeh où l'étudiant peut choisir ses maîtres?

- Oui, je suis donc allé étudier avec les meilleurs profs de la hozeh pour apprendre les sciences islamiques à la manière classique. Le plus important fut Montazeri ${ }^{8}$, auprès de qui j'ai appris le feqh. Tant qu'il était le dauphin du guide ${ }^{9}$, je suis allé une seule fois à son cours, car il était très bondé et beaucoup de personnalités officielles venaient de Téhéran avec des voitures du dernier modèle, seulement pour se montrer, sans objectif scientifique. Il y avait trois salles : le professeur se tenait dans la première et dans les autres, le cours était projeté en circuit fermé. Pour moi, ce n'était pas un cadre scientifique, et j'ai préféré écouter ses cours sur cassettes, et les transcrire. Après qu'il a été démis, à partir de 1989, j'ai assisté à son cours de manière assidue. Je me souviens que, le premier jour, j'ai vidé mes poches avant de m'y rendre et demandé à mon épouse de me pardonner si je ne revenais pas parce que, à chaque moment, on pouvait être arrêté. L'audience était tombée à $10 \%$ de ce qu'elle était avant, elle ne remplissait même pas une salle, aussi je pouvais confortablement poser des questions.

J'ai aussi pris des cours de feqh et d'osul, pendant huit ans, avec Javâd Tabrizi, qui avait étudié à Najaf avec Kho'i, ${ }^{10}$.

J'ai étudié le feqh avec sayyed Mohammad Ruhâni, aujourd'hui décédé qui, s'il était vivant, aurait un niveau scientifique plus élevé que sayyed Sistâni, à Najaf ${ }^{11}$. En fait, il était considéré comme le meilleur disciple de Kho'i ; en osul, c'était le plus fort. Mais au début, je

\footnotetext{
${ }^{8}$ Quand il n'utilise pas les titres en usage pour désigner un religieux (comme sayyed, ou ayatollâh), Mohsen Kadivar leur octroie celui de âghâ, littéralement, «monsieur ». Nous ne l'avons alors pas transcrit ici, pour éviter les lourdeurs et les répétitions.

${ }^{9}$ Hossein Ali Montazeri fut désigné par Khomeini comme son successeur, décision entérinée en 1985 par l'assemblée des experts. Suite à ses critiques publiques des pratiques du gouvernement, Montazeri fut forcé de démissionner en 1989, puis placé en résidence surveillée, à Qom ; celle-ci a été renforcée en 1997 après qu'il a critiqué l'autorité du guide Ali Khamenei. Il a été libéré en 2003. On trouvera un entretien avec lui dans Abdo, G., « ReThinking the Islamic Republic... », Middle East Journal, 55/1 (hiver 2001), pp. 9-24

${ }^{10}$ Expulsé de Najaf, où il s'était installé, peu après la révolution iranienne, en 1979, Javâd Tabrizi était devenu l'un des marja' de la hozeh de Qom avant de décéder le 20 novembre 2006 à l'âge de 82 ans. Kho'i, qui était contre la politisation des clercs, fut jusqu'à sa mort en 1992 le marja' le plus suivi dans les mondes chiites, et le rival de Khomeini.

${ }^{11}$ Mohammad Ruhâni fut parmi les marja' de Qom qui refusa la marja'iyat de Khamenei, en 1995. Cf. Buchta, W., Who rules Iran, 2000, p. 94.
} 
n'ai pas eu le courage d'aller à ses cours... J'étudiais alors la philosophie, la gnose, et l'exégèse avec l'ayatollâh Javâdi-Âmoli ${ }^{12}$, celui qui est connu aujourd'hui, dont j'étais alors très proche. Quand je l'ai consulté à ce sujet, il m'a interdit de participer au cours de Ruhâni parce que celui-ci était réputé pour être contre la révolution. Toutefois, les deux dernières années, j'ai passé outre et je suis allé à son cours de $f e q h$, car à ce moment-là il ne donnait plus de cours d'osul. Ensuite, il mourut et j'en fus très affecté.

J'essayai de m'initier aux approches de différentes écoles de $f e q h$ - nous avons des écoles variées, ce que les étudiants, souvent, ne prennent pas en considération. L'une d'entre elles était celle de l'ayatollâh Borujerdi qui avait été le maître de Montazeri et de Khomeini, entre autres, et l'unique marja' de son époque ${ }^{13}$. J'avais choisi Montazeri pour deux raisons : il était le meilleur disciple de Borujerdi et avait publié ses taqrirât du cours du maître, avec l'appui de celui-ci ${ }^{14}$. Quand j'assistai à ces cours, j'avais l'impression que c'était comme si Borujerdi lui-même enseignait. En outre, il avait été un élève particulier de monsieur Khomeini. Ainsi, les points que je voulais apprendre de Khomeini, je pouvais le faire à travers lui. En fait, pour moi, Montazeri était le représentant de la doctrine de Qom.

Je sentais par ailleurs que je devais apprendre celle de Najaf, mais c'était au temps de Saddam et je ne pouvais pas m'y rendre. Donc, je suis allé étudier auprès de oulémas qui avaient vécu à Najaf et étaient revenus à Qom, comme sayyid Ruhâni, et Javâd Tabrizi, un proche de l'ayatollâh Kho'i. Cela signifie que quand j'allais à ses cours, c'était comme si j'étais assis en face de Kho'i, qui était considéré en Iran comme le plus fameux représentant de l'école de Najaf dans la pensée chiite. Aussi, pendant mon cursus de $k$ harrej ${ }^{15}$, j'ai étudié à fond tous ses livres. J'ai donc eu trois maîtres, Borujerdi, Khomeini, et Kho'i, dont j'ai suivi les méthodes.

Il y avait aussi l'école de Mohammad Bâqer Sadr, qui fut assassiné par Saddam en 1980, avec sa sœur. C'était un génie. J'ai étudié son œuvre à Qom, avec deux de ses disciples : sayyid Kâzem Hâ'eri et Mahmud Hâchemi Shahrudi ${ }^{16}$. J'ai passé quatre ans avec le premier, qui enseigne les $o s u l$ en arabe. Aujourd'hui, il est devenu célèbre à cause du groupe de Moqtada Sadr, qui se réfère à lui, mais il n'a pas de contact avec eux. J'ai étudié un an avec le second, aujourd'hui chef du judiciaire en Iran. C'était au début de mon cycle de khârej, il enseignait en arabe car il n'était pas capable de le faire en persan. C'était quelqu'un d'intelligent, comme

\footnotetext{
12 'Abdollâh Javâdi-Amoli fait partie des oulémas qui soutiennent le gouvernement.

${ }^{13}$ Hossein Borujerdi fut le grand marja' de son temps. Sa mort, en 1961, laissa un vide et provoqua une réflexion des milieux cléricaux sur la marja 'iyat. Cf. Lambton, A., «A Reconsideration of the Position of the Marja' al-taqlîd and the Religious Institution », Studia Islamica, 20 (1964), pp. 115-135.

${ }^{14}$ Il s'agit des notes que prend l'étudiant lors du cours du maître, qu'il transcrit mot à mot et publie, avec l'accord de celui-ci. Le livre fait d'autant plus référence, dans la matière concernée, que le maître et le disciple sont reconnus comme savants.

${ }^{15}$ Troisième cycle du cursus de la hozeh.

${ }^{16}$ Tous deux sont de l'école de Najaf et vivent aujourd'hui en Iran, où ils soutiennent le gouvernement. Kâzem Hâ'eri enseigne toujours à Qom, où il a beaucoup d'étudiants. Mahmud Hashemi Shahrudi, ancien président de l'ASRII (assemblée supérieure de la
} 
Kâzem Hâ'eri, mais il était très occupé par la politique et je trouvais qu'il ne consacrait pas assez de temps aux questions scientifiques. C'est pourquoi je n'assistais qu'à une heure de son cours et, après, je me rendais à celui de sayyid Kâzem.

Enfin, j'ai étudié les osul avec Vahid Khorâsâni pendant une courte période. Son cours est considéré comme le plus prisé de la hozeh ${ }^{17}$. D'autres cours de droit islamique ne m'ont pas impressionné, tels ceux de Fâzel [Lankarâni] et Makârem [Nâser Makârem Shirazi]. J'ai étudié la science des rijâl ${ }^{18}$ avec Musa Shubairi [Zanjâni] qui est aujourd'hui un marja' et, comme je l'ai dit, la philosophie, la gnose ('erfân) et l'exégèse coranique ; on trouvait peu d'étudiants à Qom qui étudiaient l'exégèse pendant dix ans. J'ai étudié les asfâr ${ }^{19}$ avec l'ayatollah Javâdi-Âmoli : une partie en classe et l'autre moi-même, parce que le cours sur les asfâr dure vingt ans, et je n'ai pas eu la patience... Je suis allé chercher des cassettes, je les ai écoutées, et j'ai posé mes questions au professeur. J'ai procédé de la même manière pour la gnose.

Je suis resté dix-sept ans à Qom. Dès la deuxième année de mes études, je me suis mis à enseigner. Chaque jour, je donnai deux leçons et en prenais trois. Je ne pouvais avancer rapidement car à Qom, il y a beaucoup de jours fériés, à toute occasion, et la moitié de l'année est chômée. En plus, pendant la période restante, l'étudiant doit aller faire du tablîgh dans d'autres villes. Moi, je n'y allais pas, j'étudiais. Ainsi, quand la hozeh était en vacances, je commençais mon deuxième cursus, en travaillant avec des cassettes.

J'ai terminé en 1997. Avant mon départ, mon maître l'ayatollah Montazeri, m'a octroyé une ejâze (licence) d'ejtehâd, dont on peut dire que c'est le plus haut diplôme ...

- Accorde-t-on encore ce type d'ejâze?

- Oui, mais c'est rare. On donne surtout des licences de transmission, ou bien un autre type de licence, stipulant que son titulaire maîtrise les affaires relatives à la hisbiyya et les questions financières, c'est-à-dire qu'il peut gérer l'argent du khoms et de la zakât. On me l'a accordée aussi, mais je ne l'ai jamais utilisée. En outre, j'ai terminé mes études alors qu'un examen écrit était organisé, pour la première fois, à la hozeh. Je fus reçu premier et trois ayatollâhs me donnèrent un prix : le guide de la révolution Khomeini, l'ayatollah Golpâyegâni ${ }^{20}$ qui était le plus âgé dans la hozeh, et l'ayatollah Montazeri.

Tout au long de mon cursus en sciences religieuses, j'ai été préoccupé par une grande question : est-ce que nous pouvons avoir des sciences humaines islamiques ou non ? Cette question, nouvelle constituait le fondement de la révolution culturelle, selon laquelle il fallait

révolution irakienne en Iran), a été nommé à la tête du judiciaire iranien en 1999. Sur son parcours, cf. Luizard, P. J., La question irakienne, Fayard, 2002, p. 270.

${ }_{7}^{7}$ Les cours de Vahid Khorasâni sont toujours très appréciés des étudiants aujourd'hui, et ils s'y rendent en nombre.

${ }^{18}$ La science des rijâl (hommes) consiste en l'étude des chaînes de transmetteurs de hadîth.

${ }^{19}$ Il s'agit de Kitâb al-asfâr al-arba'a (Le livre des quatre voyages), de Mollâ Sadrâ Shirâzi. 
islamiser toutes les sciences. Toutefois, ceux que j'ai interrogés à ce sujet ne sont pas parvenus à me convaincre, et je me suis dit que je devais y réfléchir par moi-même. Au bout d'une dizaine d'années, je suis arrivé à la conclusion que les sciences humaines n'étaient ni islamiques, ni non islamiques. C'est pour cela que j'ai décidé de continuer ma formation à l'université en parallèle à celle de la hozeh.

\section{- Comment êtes-vous arrivé à cette conclusion?}

- Le résultat auquel je suis arrivé est que la science est la science. La géographie n'est à personne. La science n'appartient pas à la religion. Je ne peux pas nier l'influence de la religion sur la science, mais déclarer la priorité de la religion, c'est s'éloigner de la voie de la science. C'est pourquoi j'ai pensé qu'il faut d'abord comprendre une science correctement et après, envisager les relations qu'elle entretient avec une religion.

Ce qui m'a amené à cette conclusion, c'est que, après dix ans à la hozeh, je pouvais saisir seul, dans une certaine mesure, les sciences islamiques. C'est dire que, par la pratique, j'étais arrivé à un point où je n'étais plus le muqallid d'un autre. Je voyais ce qu'étaient le feqh et les sciences islamiques, l'effet qu'ils pouvaient avoir sur les autres sciences.

Il faut rappeler que dans la première décennie de la révolution, on insistait sur le potentiel du feqh; on disait qu'il pouvait résoudre toutes les questions. J'avais alors terminé le cycle du $s^{2} t^{21}$, et j'en étais arrivé à penser que, globalement, on peut extraire du feqh les sciences politiques, les sciences économiques et de la gestion. À cette époque, j'étudiais très peu hors de la hozeh. En fait, je ne sortais pas de Qom, hormis pour rendre visite à mes parents, ou pour aller sur le front, parmi les soldats.

Ma décision de m'inscrire à l'université coïncida avec la mort de Khomeini, mais je fus surtout poussé à la prendre par ma volonté d'accroître mes connaissances. J'ai ensuite intégré le cycle du khârej, le dernier, et ce fut une bonne opportunité pour moi. Un étudiant qui fréquente la hozeh avec une question en tête trouve dans ce milieu quelque chose que les autres n'y trouvent pas. Parfois, quand je posais une question, assis parmi les étudiants, je sentais qu'ils ne la comprenaient pas. Alors j'intervenais moins.

\section{- Qu'avez-vous étudié à l'université de Qom,?}

- L'intitulé exact de la discipline dans laquelle je me suis inscrit et j'ai soutenu mon doctorat, en 1999, était: philosophie islamique, section théosophie (hekmat) transcendantale. Nous avons trois écoles en philosophie islamique : l'école péripatéticienne (mashshâ'i), celle de la théosophie « orientale » (eshrâqi) ${ }^{22}$, et la théosophie transcendantale, aujourd'hui la branche

\footnotetext{
${ }^{20}$ L'ayatollâh Mohammad Reza Golpâyegâni fut considéré comme le grand marja ‘ à la mort de Khoi, en 1992, mais il décéda lui-même peu de temps après, en décembre 1993.

${ }^{21}$ Il s'agit du deuxième cycle du cursus de la hozeh, qui consiste à assimiler les ouvrages de base écrits par les oulémas, particulièrement en droit islamique.

${ }^{22}$ Mohsen Kadivar fait état ici de la classification qui a court en Iran. D'après l'intitulé de ses cours, l'école péripatéticienne se réfère à Avicenne (m. 1037); la théosophie orientale à
} 
la plus importante, qui fut fondée par Mollâ Sadrâ Shirâzi. Après lui, vécut 'Ali Modarres Tehrâni, dont Morteza Motahhari et Jalâl al-Din Ashtiyâni ${ }^{23}$ ont dit que les travaux était très novateurs. J'ai édité ses écrits en trois volumes et, dans ma thèse, j'ai analysé sa pensée et l'ai comparée à celle de Mollâ Sadrâ pour en montrer la nouveauté.

- Comment avez-vous été amené à écrire sur la philosophie politique ?

- C'est une autre histoire, puisque ma thèse portait sur la métaphysique et n'avait aucun lien avec la politique. À l'époque de la fin de la guerre, j'ai commencé à avoir des doutes, à cause du conflit qui avait éclaté entre Khomeini et Montazeri. Il s'agissait de principes théoriques, de deux visions politico-religieuses, pas seulement d'une question de goût politique. Ce fut une affaire sérieuse, qui coûta la vie à 3000 personnes, peut-être. Des individus appartenant l'organisation des Mudjahidin du peuple avaient tenté de renverser la République islamique, dans une opération préparée en coopération avec Saddam Hussein. Les responsables furent arrêtés, mais aussi un grand nombre de membres de l'organisation qui n'avaient pas participé à l'opération. À ceux-là, on demanda s'ils étaient prêts à tuer leurs amis, pour vérifier qu'ils avaient bien changé d'opinion en prison ; si c'était le cas, on les relâchait, sinon, ils étaient punis. Ce fut une rude épreuve. Montazeri, qui était alors le successeur du guide, s'opposa à ce procédé, disant qu'il n'avait aucune base légale (shar'i). Selon lui, on pouvait punir ceux qui avaient participé à cette opération, mais pas condamner leurs sympathisants à mort. Or, il est clair que l'autorisation avait été demandée à Khomeini sur cette question. Montazeri, lui, défendait ainsi le meurtrier de son propre fils, qui avait été tué quelques années auparavant dans un attentat à la bombe au Parti de la République islamique ${ }^{24}$. Cela montre la grandeur du personnage, de défendre des gens qui pensaient la même chose que ceux qui avaient tué son fils.

Ensuite, Montazeri a commencé à critiquer Khomeini en disant que cette méthode d'administration du pays n'était pas correcte et, au cours des dernières années de la guerre, il déclarait qu'il fallait y mettre fin dès que possible. De toutes façons, il y avait une divergence d'opinion entre eux et, pour moi, la mise à l'écart de Montazeri a soulevé des questions. C'est pourquoi j'ai gardé le silence et je me suis mis à étudier la philosophie politique chiite, sur laquelle il n'y avait rien d'écrit. Pendant cinq ans, j'ai étudié le sujet de velâyat-e faqih très sérieusement, en détail, en partant des premiers ouvrages de feqh, de l'époque du cheikh Saddûq.

- Vous n'alliez pas à l'université, vous lisiez seul ?

- Oui, c'était une recherche personnelle. La plupart de ces livres étaient lithographiés, n'avaient pas de tables de matières et étaient difficiles à déchiffrer. Tout était dans le

Sohravardi, (m. 1191) et la théosophie transcendantale à son disciple Mollâ Sadrâ (m. 1640). Sur ces questions, cf. Corbin, H., Histoire de la philosophie islamique, 1986.

${ }^{23}$ Celui-ci avait collaboré avec Henry Corbin, et enseignait la philosophie à Mashhad. Il est décédé en 2005.

${ }^{24}$ Mohammad Montazeri, considéré comme le $n^{\circ} 2$ du parti après Beheshti, fut tué avec ce dernier dans cet attentat, en 1980. 
désordre. Les études sur la politique, chez les chiites, ne sont pas développées comme chez les sunnites, qui ont les ahkâm sultâniyya. J'ai d'abord publié un article que le journal Jomhuri eslâmi, qui appartient à Khamenei, a critiqué. Il se transforma ensuite en livre, dont le premier volume est Nazariyat-e dolat dar feqh-e shi'i (Théories de l'État dans le droit islamique chiite), traduit en arabe, et le second Hokumat-e velâyi (Le gouvernement selon le principe de velâyat-e faqih). Depuis, je donne des cours en sciences politiques au niveau du doctorat et ces livres sont devenus des manuels enseignés à l'université cheikh al-Mofid ${ }^{25}$.

- Vous-même avez-vous enseigné à l'université cheikh al-Mofid ?

- Oui, de 1993 à 1996, j'ai été le recteur du département de philosophie. J'y ai aussi enseigné d'autres matières et j'ai été membre du comité scientifique. Maintenant, ici, à l'université Tarbiyat-e modarres, j'enseigne au département de philosophie et je n'ai plus le temps de donner des cours ailleurs.

- Vous n'enseignez plus à la hozeh?

- Non. Lorsque je que je suis venu à Téhéran, les étudiants qui assistaient à mes cours ont subi des pressions. Chaque année, les étudiants de la hozeh, ont besoin d'une attestation de leur professeur pour être dispensés du service militaire. Quand ils montraient ma signature, cela leur causait un problème...La pression à Qom est plus forte qu'à Téhéran. C'est pourquoi, pratiquement, j'ai dû m'exiler à Téhéran. Maintenant, je ne fais plus que de la recherche dans le cadre de la hozeh, sur les droits humains, d'autres questions relatives aux sciences religieuses, et je poursuis mon travail sur la philosophie politique du chiisme.

- Comment vous êtes-vous intéressé aux droits humains ?

- La question des droits humains était couramment débattue, l'islam était accusé car dans ce domaine, il n'et pas convaincant. Les études disponibles n'étaient que défensives et c'étaient des discussions polémiques où certains soutiennent que l'islam a des aspects positifs et que cela doit être ainsi. Lorsque j'ai vérifié, je me suis rendu compte que ce n'était pas comme ça. Si nous voulons étudier de façon impartiale certains préceptes, il faut se dire qu'ils ne sont pas immuables. J'ai commencé par un cas simple, dont j'ai parlé dans un colloque scientifique international organisé à l'université cheikh Mofid, en présentant un exposé sur l'esclavage dans l'islam contemporain. Ensuite, j'ai montré que, en comparaison avec la déclaration universelle des droits de l'homme, les droits islamiques, le feqh islamique posait problème sur plusieurs points, et je me suis donné pour objectif de les étudier un par un, sérieusement et en détail. J'ai fait toutes les analyses, ce qui constituait la partie la plus facile. Maintenant, il me faut du temps. J'ai donné une série de cours sur les droits de la femme dans le Coran, auquel plus de cent femmes ont participé.

- À l'université ?

\footnotetext{
${ }^{25}$ C'est une université islamique moderne, réformée.
} 
- Non, au parti Moshârekat ${ }^{26}$, parce que nous avons des problèmes à enseigner certains sujets dans les classes. Quand j'ai commencé mes cours, des étudiants s'étaient plaints du fait que j'avais critiqué Mollâ Sadrâ et ainsi semé la confusion dans leur religion. En tant que professeur, si je ne peux pas critiquer Mollâ Sadrâ en cours, où puis-je le faire ? À l'université, j'enseigne seulement les sujets qui ont été approuvés par le ministère de l'Education. Mais à l'extérieur, je suis plus libre. J'ai aussi donné des conférences sur les droits des enfants. Ce sont des terrains difficiles. Mes articles ont été vivement critiqués, on a écrit que j'ai commis une bed'at (innovation blâmable), que je suis hors de la religion, que j'ai trahi, etc. Ces critiques ont été déposées auprès du pouvoir judiciaire et constituent un dossier contre moi. Les références de ces articles sont sur mon site, à la rubrique « critique des œuvres ». On reproche à mes écrits d'être contre l'islam traditionnel (eslâm-e sonnati), ce que je sais.

- Qu'est-ce que cela signifie?

- L'islam des intellectuels a un feqh spécial, et c'est pour cette raison que nous sommes considérés comme dangereux. Ils disent que ce n'est ni de la religion, ni du feqh. Nous disons que nous avons de la religion, du feqh, et des valeurs morales, et que ce dont ils parlent ne fait pas partie de notre religion, de notre $f e q h$, de notre morale. Notre gouvernement est un gouvernement feqhi (fondé sur le feqh) et il le revendique. Je dois donc le critiquer de ce point de vue. Ils craignent qu'une autre lecture du feqh que la leur soit présentée. Si vous leur dites que ce n'est pas du feqh, cela ne pose pas de problème. Si vous leur dites que c'est du feqh, ils vous m'accusent d'éliminer la charia. Je dis non.

La différence entre nous et les conservateurs est qu'ils veulent nous faire revenir au temps du prophète, ce qui nous ramène en arrière. Moi, je dis qu'il faut faire venir le prophète à notre époque. Mais si nous l'amenions à notre époque, est-ce qu'il viendrait en chameau? Absolument pas. Il viendrait en voiture. Aujourd'hui si le prophète venait et que les droits humains étaient dans la situation que nous connaissons, les rejetterait-il ? Je pense que nous devons comprendre le prophète en notre temps.

Sur les droits humains, j'ai aussi écrit un article sur l'apostasie et la liberté de conscience, lorsque Hâshem Aghâjari et Hasan Yosefi Eshkevari étaient accusés de kufr (incroyance) ${ }^{27}$. Enfin, mes recherches sur les droits humains m'ont amené à m'intéresser à la philosophie du

\footnotetext{
${ }^{26}$ Créé en 1999, Hezb-e moshârekat regroupe des partisans de Mohammad Khâtami ; il est dirigé par son frère.

${ }^{27}$ Hâshem Aghâjeri fut arrêté et accusé d'apostasie en 2002, après un discours critique sur la hiérarchie cléricale dans le chiisme, notamment le concept de taqlîd. Condamné à mort, il fut gracié par le Guide Ali Khamenei et relaxé en 2004. Cf. Mir-Hosseini, Z., et Tapper, R., Islam and Democracy in Iran, 2006, p. 179. Pour H. Y. Eshkevâri, cf. le chapitre le concernant dans le présent ouvrage. L'article en question, « The freedom of thought and religion in islam » est sur le site de Mohsen Kadivar ; une version courte est publiée dans Baktiari, B. et Norton, A. R., « Voices within Islam : Four Perspectives on Tolerance and Diversity », Current History, janvier 2005, p. 43-44.
} 
droit pénal, que j'ai enseignée à l'université. Les problèmes sociaux m'ont aussi entraîné sur cette voie, j'ai par exemple écrit sur le droit pénal des femmes.

- Tout cela en fondant vos démonstrations sur les sciences religieuses ?

- Oui, en utilisant les termes de $f e q h$, en tirant profit de nos traditions, comme celle de Achoura, qu'il est important de marquer, chaque année. Je m'appuie sur les piliers des droits humains en islam voulant que le droit des gens pèse plus que le droit de Dieu, parce que Dieu pardonne l'atteinte à son droit, mais pas l'atteinte au droit des hommes. En Iran, où la société est très religieuse, aucun changement ne peut survenir s'il n'est pas clairement posé par rapport à la tradition religieuse.

Les lecteurs les plus nombreux de mes livres sont les oulémas et les étudiants de la hozeh. Je vais à Qom une fois par mois, et y rencontre mes maîtres, notamment Montazeri, et des oulémas.À chaque fois, je leur parle de quelque chose de nouveau. Je n'ai plus d'étudiants à la hozeh, mais ils me contactent par téléphone ou par e-mail, ils lisent mes articles, les discutent en cours et m'écrivent leur avis.

Aujourd'hui en Iran comme ailleurs, il y a plusieurs tendances. Certains ne font que critiquer la tradition, et suivent la modernité. Ils ont abandonné la religion. Nous avons aussi les partisans de la tradition, et d'autres groupes, qui pensent que la modernité est une cause de déviance. Ils croient que la seule voie est celle de la revivification absolue de notre tradition. Pour ma part, je crois qu'i y a des points très importants dans la tradition pur la vie de l'homme moderne. Il en est de même pour la modernité. Je crois que la tradition et la modernité ont besoin l'une de l'autre, et qu'il faut les critiquer toutes les deux.

- Votre dernier livre porte sur Akhund Khorasâni ${ }^{28}$; pourquoi ce choix ?

- Ce livre rassemble des documents et des lettres de Khorasâni, en arabe, que j'ai consignés et dont je présenterai une analyse dans le second volume, en préparation. Avant le livre, j'avais publié un article, suite à une série de séminaires auxquels j'avais participé à l'université Doshisha, à Kyoto, au Japon. On m'avait demandé d'intervenir sur la hozeh de Qom, mais j'avais préféré parler de celle de Najaf, et du grand savant chiite qui y résidait il y a cent ans, Akhund Khorasani ${ }^{29}$. Il avait renversé Mohammad Ali Shah Qâjâr comme Khomeini a renversé Mohammad Reza Shah, ensuite. Ils ont mené le même combat, mais leurs écoles de pensée s'opposent car Khorasâni ne croit en en aucune version ni aucune forme de velâyat-e faqih $^{30}$. Ainsi, après l'occultation du douzième imam, c'est selon lui aux gens de gouverner. Ni le prophète ni l'imam ne détenaient la guidance absolue (velâyat-e motlaq), elle revient à

\footnotetext{
${ }^{28}$ Mollâ Kâzem Khorasâni (m. 1911) fut un marja', un grand maître en osul et un fervent partisan du mouvement constitutionnaliste en Iran.

${ }_{29}$ Après ce séminaire à Kyoto, en mars 2004, M. Kadivar participa à une table ronde à l'université de Tokyo. Le texte de sa contribution « Political Innovative Ideas and Influence of Molla Mohammad Kazim Khorasani » est disponible sur son site, en anglais, et a été publié.

${ }^{30}$ Il s'agi là d'une lecture de la pensée de Khorasâni puisque, bien sûr, le concept de velâyat-e faqih n'existait pas.
} 
Dieu seul. Lorsque j'ai écrit Nazariyât-e dolat (Les théories de l'État), je n'ai pas pris ce fait en considération, je m'intéressais à l'époque à l'école de Kho'i, mais Khorasâni est plus fort. Lorsque j'ai rapporté cela à mon maître Montazeri, il a été surpris ; j'en ai parlé à d'autres grands oulémas, à Qom, qui ne connaissaient Khorasâni que pour son œuvre sur les osul. Si je ne peux pas accepter tout ce qu'il écrit, car c'est une vision politique qui date de cent ans, son discours est supérieur à ceux des oulémas d'aujourd'hui, à Qom ou à Najaf. Kho'i n'avait pas les qualités de Khorasâni ; il n'était pas militant.

J'ai eu des difficultés à trouver un éditeur pour ce livre, et il est resté un an en attente d'autorisation de publication, à la censure. Ensuite, l'introduction que j'ai écrite a été critiquée dans des revues ${ }^{31}$.

Aujourd'hui, je travaille aussi en théologie (kalâm), sur l'imamat. J'ai montré que dans les cinq premiers siècles de l'islam, deux interprétations coexistaient. L'une créditait les imams der la 'isma (infaillibilité) et de la connaissance du ghayb (ce qui est caché aux hommes) L'autre les considérait comme des hommes ordinaires, seulement dotés de plus de savoir que les autres. La première interprétation, alors minoritaire, l'a finalement emporté ; l'autre a été oubliée ${ }^{32}$. Par ailleurs, en partant d'une classification des croyants, et en m'appuyant sur trois savants (cheikh Tusi, al-Shahid al-Thâni, Morteza Ansâri ${ }^{33}$ ), j'ai établi qu'il existe une sorte de pluralisme dans l'islam : si un individu professe une autre croyance que l'islam, personne ne peut le lui reprocher. Cela change tout sur la question de l'apostasie ${ }^{34}$.

Propos recueillis par Sabrina Mervin, juillet 2004 et février 2007

Traduction Mohammad Tehrâni et Cyril Mercier

Bibliographie

Abdo, Geneive

«Re-Thinking the Islamic Republic: A 'Conversation' with Ayatollah Hossein 'Ali Montazeri », Middle East Journal, 55/1 (hiver 2001), p. 9-24.

Abrahamain, Ervand,

Compte-rendu de lecture de Andishe-e siyâsi dar islam, Islamic Law and Society, 8/2, 2001, p. 295-298.

Baktiari, Bahman et Norton, Augustus Richard, «Voices within Islam : Four Perspectives on Tolerance and Diversity », Current History, janvier 2005, p. 37-45.

\footnotetext{
${ }^{31}$ Cette introduction est contre velâyat-e faqih.

${ }^{32}$ Article publié dans la revue Madrasa, cf. "Qerâ'at-e faramush shode...» (Les interprétations oubliées), vol. 1, n³, mai 2006, pp. 92-102.

33 Tous trois ont marqué l'histoire des doctrines chiites. Le cheikh al-Tusi (m. 1067); alShahid al-Thâni (le deuxième martyr) est le surnom de Hasan b. Zayn al-Din al-'Âmili, un savant du Jabal 'Âmil (m. 1602); Morteza Ansari (m. 1864) fut un maître de Mollâ Kâzem Khorasâni, à Najaf.

34 « Tabaqebandiâe e 'teqâdat-e dini » catégorisation des croyances religieuses), Madrase, vol. $2, n^{\circ} 5$, février 2007, pp. 21-26.
} 
Buchta, Wilfried,

Who rules Iran? The Structure of Power in the Islamic Republic, Washington Institute for Near East Policy-Konrad Adenauer Stiftung, Washington, 2000.

Kadivar, Mohsen,

Andishe-e siyâsi dar islâm (La pensée politique en islam), 2 vol., Nashr-e nay, Téhéran.

Vol. 1 : Nazariyehâ-ye dowlat dar feqh-e shi'i (Les théories de l'État dans le droit islamique chiite), 1998 ; vol. 2 : Hokumat-e velâ'i (Le gouvernement fondé sur velâyat-e faqih), 1999.

Traductions en arabe du premier volume :

Nazariyyât al-hukm fî al-fiqh al-shî‘î: buhûs fî̀ wilâyat al-faqîh, dâr al-Jadîd, Beyrouth 2000.

Nazariyyât al-dawla fî al-fiqh al-shî‘î (ma'a muqaddima naqdiyya) traduction dr sheikh Muhammad Shuqayr, dâr al-Hâdî, Beyrouth, 2004.

« Velayat-e faqih and Democracy » www.kadivar.com

«Political Innovative Ideas and Influence of Molla Mohammad Kazim Khorasani», JAMES International workshop Changing knowledge and authority in Islam, the University of Tokyo, marth 26, 2004. Annals of Japan Association for Middle East Studies (ajames), $\mathrm{n}^{\circ} .21-1$, septembre 2005, Tokyo, p.59-73.

Farzin Vahdat

«Post-revolutionary Discourse of Mohammad Mojtahed Shabestari and Mohsen Kadivar : Reconciling the Terms of Mediated Subjectivity. Part II: Mohsen Kadivar », Critique, 17 (2000), p. 135-157.

Lambton, Ann K. S.

"A Reconsideration of the Position of the Marja ' al-taqlîd and the Religious Institution", Studia Islamica, 20 (1964), p. 115-135.

Luizard, Pierre-Jean, La question irakienne, Fayard, Paris, 2002.

Mottaghi, Mohsen

Iran : les nouveaux intellectuels musulmans, thèse de doctorat inédite, sous la direction de Farhad Khosrokavar, EHESS, 2004.

Sadri, Mahmood

«Sacral Defense of Secularism: The Political Theologies of Soroush, Shabestari and Kadivar », International Journal of Politics, Culture and Society, 15/2, hiver 2001, p. 257270. 